\title{
Conformación de una cartera de inversión óptima de cultivos agrícolas para México
}

\author{
Miguel Ángel Díaz-Carreño \\ RAFAEL JuÁREZ-TOLEDO \\ María del Carmen Gómez-Chagoya*
}

\section{Resumen}

Este artículo presenta una cartera de inversión óptima de cultivos agrícolas para México a partir del enfoque teórico de conformación de portafolios de inversión que introdujo Harry Markowitz. Se emplearon tres grupos de cultivos: cereales, frutales y hortalizas. La cartera óptima se integró con cultivos de los tres grupos a partir de los principios de diversificación del portafolio, también se incluyeron aquellos cultivos que presentaron mayor frecuencia de correlación negativa de sus rendimientos con el resto de los cultivos considerados. La cartera óptima se conforma con cultivos de sorgo, trigo, aguacate, mango, manzana, naranja, piña, chile verde, papa y sandía. Esta cartera es eficiente en el sentido de que, por un lado, permite maximizar el nivel de los rendimientos esperados y, por otro, minimizar el nivel de riesgo.

Palabras clave: cartera de inversión, cultivos agrícolas, rendimiento, riesgo.

\begin{abstract}
This paper presents a portfolio of agricultural products for optimal investment in Mexico from the theoretical framework of investment portfolio setup introduced by Harry Markowitz. Three types of crop were used: cereals, fruit and vegetables. The optimal portfolio was integrated with crops of the groups mentioned above based on the principles of portfolio diversification. We have also included those crops that showed greater frequency of negative correlation in their yields with respect to the rest of the crops considered. The optimal portfolio includes: sorghum, wheat, avocado, mango, apple, orange, pineapple, green chilli, potato and watermelon. This portfolio is efficient in the sense that, on the one hand it allows maximising the level of expected returns, and on the other hand, minimising the risk levels.
\end{abstract}

Keywords: Portfolio of investment, agricultural cultures, yield, risk

* Facultad de Economía de la Universidad Autónoma del Estado de México. Correose: madiaz@colpos.mx, rjtoledo@yahoo.com.mxy cgchagoya@yahoo.com.mx. 


\section{Introducción}

A partir de la entrada en vigor del Tratado de Libre Comercio de América del Norte (TLCAN), en el sector agrícola mexicano se intensificó el fenómeno de reconversión de cultivos de aquellos considerados menos rentables, como es el caso de algunos cereales, a otros con mayor rendimiento, como ciertos frutales y hortalizas.

Adrián González (2002: 93) señala que la liberalización comercial y la apertura del sector financiero a los flujos de capital extranjero cambiarán el patrón de cultivos y la distribución geográfica de las actividades agrícolas de México; además de que la superficie cultivada con granos básicos (maíz, frijol, trigo y arroz) se reducirá considerablemente después de que se elimine la totalidad de las barreras proteccionistas y desaparezcan todas las transferencias y apoyos compensatorios, los cultivos básicos perderán importancia relativa dentro del producto interno bruto (РIB) agrícola, al tiempo que crecerá la de frutales y hortalizas.

De acuerdo con Quintana (2003: 8), en la geografía agrícola mexicana se ha dado un fuerte cambio: mientras se reduce la superficie dedicada a granos básicos y oleaginosas, hay un dinamismo significativo en el crecimiento de frutas, hortalizas y cultivos forrajeros orientados a los mercados externo e interno más rentables. Así, la superficie hortícola aumentó en 6\% y la frutícola, en $21 \%$.

Esta tendencia tiene su fundamento en una ventaja comparativa de México respecto de Estados Unidos para producir frutales y hortalizas, y no cereales (Suárez, 1995: 74); sin embargo, Martínez y Díaz (2005: 75) argumentan que producir frutales y hortalizas es más riesgoso que cultivar cereales.

En México, la superficie dedicada a siembras agrícolas ascendió a más de 20 millones de hectáreas en promedio durante el periodo 1994-2003 (Sagarpa, 2005): 67\% se dedicó a granos mientras que el cultivo de hortalizas sólo ocupó 3.5\% de esa superficie, y en el caso de los frutales, se empleó únicamente $6 \%$. No obstante lo anterior, el cultivo de granos aporta solamente $36 \%$ del valor total de la producción, en tanto que los frutales y hortalizas contribuyen con 18 y 16\%, respectivamente. Dichos valores reflejan la importancia de estos cultivos en la agricultura del país (Siller, 2004: 04).

Ante esta disyuntiva, en la presente investigación se estudia la conveniencia de continuar con los actuales esquemas de producción agrícola a partir de un análisis de rendimiento-riesgo que 
implican los principales grupos de cultivos en México. De esta manera, el objetivo de este trabajo consiste en determinar los niveles de riesgo y rendimiento de los cereales, frutales y hortalizas, tomados éstos como portafolios, así como conformar un portafolio de inversión óptimo, considerando los cultivos de los tres grupos, a partir de la teoría de la conformación de portafolios de inversión de Markowitz (1991: 15). Es decir, se integrará una cartera de cultivos que por un lado maximice los rendimientos, ${ }^{1}$ y por otro, minimice el riesgo. ${ }^{2}$ A partir de esto se estará en condiciones de constituir una propuesta inicial en torno a una posible reconversión de cultivos la cual, sin embargo, tendría que analizarse bajo la consideración de investigaciones relacionadas que permitan disponer de los elementos necesarios para una posible toma de decisiones al respecto, así como tomar en cuenta que los resultados mostrados en este documento corresponden a una metodología en particular y a un periodo específico: 1980-2004.

Este artículo consta de tres apartados. En el primero se analiza la función de utilidad de un inversionista agrícola, la cual depende fundamentalmente del riesgo y del rendimiento que ésta genera. En seguida se describe la metodología que conforma los portafolios de inversión, la cual permite analizar el riesgo-rendimiento que existe en una inversión. Finalmente se discuten los resultados y conclusiones obtenidos destacando la propuesta de un portafolio óptimo de cultivos agrícolas para México.

\section{Riesgo y rendimiento en cultivos agrícolas}

El problema fundamental de los inversionistas es cómo distribuir su riqueza entre los múltiples activos existentes, de modo que puedan maximizar su utilidad esperada (UL $\left.{ }^{\mathrm{e}}\right)$, la cual a su vez depende del rendimiento esperado de la inversión $\left(\mathrm{r}^{\mathrm{e}}\right)$ así como del riesgo, representado por la varianza de los rendimientos $\left(\tilde{\mathrm{A}}^{2}\right)$, o bien:

$$
\begin{aligned}
& \mathrm{UL}^{\mathrm{e}}=\mathrm{UL}\left(\mathrm{r}^{\mathrm{e}}, \tilde{\mathrm{A}}^{2}\right) \\
& +-
\end{aligned}
$$

La ecuación (1) muestra que la utilidad esperada aumenta cuando se incrementa el rendimiento esperado del portafolio

${ }^{1} \mathrm{El}$ rendimiento de un cultivo agrí́cola se mide como la cantidad de producto generada por unidad de superficie cosechada en cierto periodo, por ejemplo: toneladas por hectárea (ton/ha) durante un año.

2 Medido a través de la varianza de los rendimientos. 
$\left(\partial \mathrm{UL} / \partial \mathrm{r}^{\mathrm{e}}>0\right)$ y declina cuando éste se hace más variable, esto es, cuando $\tilde{A}^{2}$ aumenta $\left(\partial \mathrm{UL} / \partial \tilde{A}^{2}<0\right)$.

En este estudio se asume que los inversionistas presentan aversión al riesgo; es decir, les interesa reducir el riesgo tanto como maximizar los rendimientos esperados.

Si los agentes no se preocuparan por el riesgo, se entendería que son neutrales a éste. Pero si así fuera, los individuos no contratarían seguros ni los inversionistas tratarían de diversificar sus inversiones financieras. Sería suficiente mantener un solo activo, el que prometiera el rendimiento más alto. Por el contrario, como los agentes contratan seguros y dedican considerable esfuerzo a diversificar sus inversiones, se concluye que el supuesto de aversión al riesgo es apropiado (Sachs y Larraín, 1994: 631).

El tema de la conformación de carteras de inversión para el sector agrícola no se ha abordado suficientemente en México, son escasos lo trabajos orientados al análisis del riesgo y rendimiento de los cultivos agrícolas a partir de éste enfoque teórico.

Martínez (1999: 119) argumenta que algunas veces se ha evaluado el riesgo en la actividad agrícola mediante métodos que no requieren del planteamiento de una función de distribución probabilística, con la implicación de sobreestimar lo riesgoso de una actividad. Además, evalúa la dispersión que presenta la estimación del riesgo obtenida empleando, por un lado, una distribución probabilística y por otro, un método libre de distribución. Dicha evaluación consistió en comparar dos métodos aplicados a una muestra de productores de trigo, en la que se aproximó al ingreso con una función de distribución beta en tres parámetros y una función de bienestar logarítmica, encontrando que la estimación de la prima de riesgo se puede reducir hasta en 1.52 veces con respecto al método conservador.

Martínez y Díaz (2005: 75) presentaron un análisis del nivel de riesgo entre los cultivos de cereales, frutales y hortalizas a partir del cálculo de la varianza de los índices de precios de dichos cultivos. En el estudio se observó que dicha varianza resulta significativamente mayor en el caso de los frutales y hortalizas respecto de los cereales cuando la metodología de cálculo del índice de precios fue la de Laspeyres, de Paasche y Fischer. En el trabajo se concluyó que la inversión en la producción de frutales y hortalizas presentaba mayor nivel de riesgo respecto del cultivo de cereales. 


\section{Metodología de la investigación}

Existen diversos procedimientos estadísticos que permiten estimar el nivel de riesgo y rendimiento para cultivos agrícolas (Sánchez, 2001: 251). Entre otros, la estimación del riesgo de los cultivos agrícolas se puede aproximar a través del cálculo de la varianza de sus índices de precios correspondientes (Martínez y Díaz, 2005: 79). Para tal fin, en este trabajo se emplea la teoría de la selección de portafolios de inversión ${ }^{3}$ introducida por Harry Markowitz (1952: 11). Su percepción más importante fue que los agentes intentan alcanzar alguna combinación óptima de riesgo y rendimiento en sus portafolios. Para llegar a esta combinación, la estrategia óptima de inversión involucra, por una parte, la diversificación del portafolio, esto es, mantener un portafolio que tiene pequeñas cantidades de un gran número de activos, ${ }^{4}$ y por otra, que en la medida que un portafolio contenga más elementos con covarianza negativa de sus rendimientos respecto del resto de los cultivos incluidos, el nivel de riesgo global de la cartera se reducirá de manera significativa sin afectar sustancialmente el rendimiento de la misma.

Markowitz (1991: 469) considera que para evaluar un portafolio y tomar decisiones en torno a éste, únicamente se requiere conocer dos elementos: el riesgo y el rendimiento medio. Diversos estudios, entre ellos el de Tobin (1958: 65), concluyen que en la mayoría de los casos los rendimientos siguen una distribución normal, lo cual permite apoyar el planteamiento de Markowitz en el sentido de que si una variable aleatoria se distribuye en forma normal, ésta se puede describir en su totalidad a partir de sus dos primeros momentos, los cuales corresponden a la media y la varianza (Serrano, 1993: 6).

A continuación se describe el procedimiento a partir del cual es posible estimar el rendimiento esperado (media), así como el riesgo (varianza) de un portafolio de inversión.

Consideremos un inversionista que posee un monto dado de recursos, $W_{0}$, y que invierte en $N$ activos distintos. La fracción del portafolio global invertida en el activo $j$ se designará como $a_{i}$; $\mathrm{j}=1,2, \ldots, N$. En términos absolutos, $\mathrm{a}_{\mathrm{j}} \mathrm{W}_{0}$ estará invertido en el activo j. Como las fracciones correspondientes a todos los activos deben sumar uno, tenemos que $a_{1}+a_{2}+a_{3}+\ldots+a_{N}=1$.

\footnotetext{
${ }^{3}$ Es común emplear como sinónimo de portafolio de inversión, cartera de inversión. En este trabajo los conceptos son equivalentes.

${ }^{4}$ En este estudio un activo corresponde a un cultivo en particular.
} 
En general, si un activo genera un rendimiento $r_{1}$, un rendimiento $r_{2}$ y así sucesivamente hasta $r_{n}$, entonces el rendimiento esperado, $r^{e}$, sobre el activo se puede obtener a partir del valor esperado de sus rendimientos, $\mathrm{E}\left(r^{e}\right)$, el cual podemos estimar mediante la siguiente expresión:

$$
r_{i}^{e}=n^{-1}\left(r_{1}+r_{2}+r_{3}+\ldots+r_{n}\right) ; I=1,2, \ldots, N
$$

donde n representa el número de retornos que genera el activo. Para obtener el rendimiento esperado del portafolio global, $r_{p}^{e}$, primero se determina el rendimiento de cada uno de los $N$ activos, de acuerdo con la expresión (2). Se les designa como $r_{1}^{e}$, $r_{2}^{e}, \ldots, r_{N}^{e}$, para los activos $1,2, \ldots, N$, respectivamente. El rendimiento esperado del portafolio $\left(r_{p}^{e}\right)$ se calcula entonces como un promedio ponderado de los rendimientos esperados de los $N$ activos que forman parte del portafolio, en el que las ponderaciones corresponden a las fracciones del portafolio invertidas en cada uno de los activos; es decir:

$$
\mathrm{r}_{\mathrm{p}}^{\mathrm{e}}=\mathrm{a}_{1} \mathrm{r}_{1}{ }^{\mathrm{e}}+\mathrm{a}_{2} \mathrm{r}_{2}{ }^{\mathrm{e}}+\ldots+\mathrm{a}_{\mathrm{N}} \mathrm{r}_{\mathrm{N}}^{\mathrm{e}}
$$

Por tanto, el rendimiento esperado de un portafolio depende al mismo tiempo de los rendimientos esperados de cada uno de los activos componentes del portafolio, como de la fracción de los recursos asignados a cada activo.

Por otra parte, es posible medir el riesgo de un portafolio a través de la obtención de la varianza de los rendimientos que genera. Primero examinemos el concepto de varianza en el nivel de un solo activo $\left(\tilde{\mathrm{A}}^{2}\right)$.

Técnicamente, la varianza de los rendimientos de un activo se define como la suma ponderada de las desviaciones cuadráticas de los rendimientos del activo respecto de su media (Mood et al., 1974: 229), el factor de ponderación es (n-1), el cual representa los grados de libertad con que se realiza esta estimación, es decir:

$$
\tilde{A}_{i}^{2}=(n-1)^{-1}\left[\left(r_{1}-r_{i}^{e}\right)^{2}+\left(r_{2}-r_{i}^{e}\right)^{2}+\ldots+\left(r_{n-} r_{i}^{e}\right)^{2}\right] ; i=1,2, \ldots, N
$$

donde $r_{i}^{e}$ es el rendimiento esperado de un activo, definido en la ecuación (2). Es común observar que en lugar de la varianza, se emplea la desviación estándar como la medida del riesgo; es decir, la raíz cuadrada de $\tilde{\mathrm{A}}_{i}^{2}$. 
Consideremos un portafolio con dos activos, 1 y 2, que tienen rendimientos esperados $\mathrm{r}_{1}{ }^{\mathrm{e}}$ y $\mathrm{r}_{2}{ }^{\mathrm{e}}$, respectivamente. Entonces, su rendimiento medio, de acuerdo con (3), será:

$$
\mathrm{r}^{\mathrm{e}}=\mathrm{a}_{1} \mathrm{r}_{1}{ }^{\mathrm{e}}+\mathrm{a}_{2} \mathrm{r}_{2}{ }^{\mathrm{e}} ; \text { donde: } \mathrm{a}_{1}+\mathrm{a}_{2}=1
$$

en tanto que la varianza de este portafolio se define en seguida (Ross, 2002: 123): ${ }^{5}$

$$
\begin{aligned}
& \tilde{A}^{2}\left(a_{1} r_{1}+a_{2} r_{2}\right)=\operatorname{Var}\left(a_{1} r_{1}+a_{2} r_{2}\right)=\operatorname{Var}\left(a_{1} r_{1}\right)+\operatorname{Var}\left(a_{2} r_{2}\right)+ \\
& 2 a_{1} a_{2} \operatorname{Cov}\left(r_{1}, r_{2}\right)
\end{aligned}
$$

donde $\operatorname{Cov}\left(\mathrm{r}_{1}, \mathrm{r}_{2}\right)$ es la covarianza de los rendimientos de los activos 1 y 2, que tienen rendimientos $r_{11}$ y $r_{21} ; r_{12}$ y $r_{22}$; y así sucesivamente hasta $r_{1 n}$ y $r_{2 n}$. Si hay $\mathrm{n}$ combinaciones posibles diferentes de resultados entonces:

$$
\begin{aligned}
& \operatorname{Cov}\left(\mathrm{r}_{1}, \mathrm{r}_{2}\right)=(\mathrm{n}-1)^{-1}\left[\left(\mathrm{r}_{11}-\mathrm{r}_{1}^{\mathrm{e}}\right)\left(\mathrm{r}_{21}-\mathrm{r}_{2}^{\mathrm{e}}\right)+\left(\mathrm{r}_{12}-\mathrm{r}_{1}^{\mathrm{e}}\right)\left(\mathrm{r}_{22}-\mathrm{r}_{2}^{\mathrm{e}}\right)\right. \\
& \left.+\ldots+\left(\mathrm{r}_{1 \mathrm{n}}-\mathrm{r}_{1}^{\mathrm{e}}\right)\left(\mathrm{r}_{2 \mathrm{n}}-\mathrm{r}_{2} \mathrm{e}\right)\right]
\end{aligned}
$$

De acuerdo con la definición de varianza en (6), es posible generalizar ésta al caso de un portafolio con $\mathrm{N}$ activos $\left(\tilde{\mathrm{A}}_{\mathrm{p}}{ }_{\mathrm{p}}\right)$. Así, la varianza del portafolio global es la suma ponderada de cada una de las varianzas de los activos componentes más un término que corresponde a la covarianza de dos activos (Sachs y Larraín, 1994: 635), esto es:

$$
\begin{aligned}
& \tilde{\mathrm{A}}^{2}{ }_{\mathrm{p}}=\operatorname{Var}\left(\mathrm{a}_{1} \mathrm{r}_{1}{ }^{e}+\mathrm{a}_{2} \mathrm{r}_{2}{ }^{\mathrm{e}}+\ldots+\mathrm{a}_{\mathrm{N}} \mathrm{r}_{\mathrm{N}}{ }^{\mathrm{e}}\right)=\mathrm{a}^{2}{ }_{1} \tilde{\mathrm{A}}^{2}{ }_{1}+\mathrm{a}^{2}{ }_{2} \tilde{\mathrm{A}}^{2}{ }_{2}+\ldots \\
& +\mathrm{a}^{2}{ }_{\mathrm{N}} \tilde{\mathrm{A}}^{2} \\
& +2 \mathrm{a}_{1} \mathrm{a}_{2} \operatorname{Cov}\left(\mathrm{r}_{1} \mathrm{r}_{2}\right)+2 \mathrm{a}_{1} \mathrm{a}_{3} \operatorname{Cov}\left(\mathrm{r}_{1} \mathrm{r}_{3}\right)+\ldots+2 \mathrm{a}_{1} \mathrm{a}_{\mathrm{N}} \operatorname{Cov}\left(\mathrm{r}_{1} \mathrm{r}_{\mathrm{N}}\right) \\
& +2 \mathrm{a}_{2} \mathrm{a}_{3} \operatorname{Cov}\left(\mathrm{r}_{2}, \mathrm{r}_{3}\right)+2 \mathrm{a}_{2} \mathrm{a}_{4} \operatorname{Cov}\left(\mathrm{r}_{2} \mathrm{r}_{4}\right)+\ldots+2 \mathrm{a}_{2} \mathrm{a}_{\mathrm{N}} \operatorname{Cov}\left(\mathrm{r}_{2,} \mathrm{r}_{\mathrm{N}}\right) \\
& +\ldots+2 \mathrm{a}_{\mathrm{N}-1} \mathrm{a}_{\mathrm{N}} \operatorname{Cov}\left(\mathrm{r}_{\mathrm{N}-1,{ }_{\mathrm{N}}} \mathrm{r}_{\mathrm{N}}\right.
\end{aligned}
$$

Es posible obtener una expresión equivalente, como en (7), para cada término de covarianza que se presenta en (8).

Un inversionista agrícola puede elegir entre diversos cultivos y conformar múltiples portafolios considerando distintas participaciones de cada uno de los cultivos. Cada combinación de cultivos en un portafolio específico podrá caracterizarse por su nivel de riesgo y rendimiento.

${ }^{5}$ La varianza de una variable aleatoria (o función de variables aleatorias) comúnmente se denota con $\mathrm{s}^{2}($.), o bien, por el término Var (.). 
Al conjunto de todos los portafolios que es posible construir con diversas combinaciones de los cultivos se le llama conjunto de portafolios factibles. Éste muestra las combinaciones riesgorendimiento que se pueden obtener mediante diferentes elecciones para el portafolio. Sin embargo, en la práctica el inversionista centra su interés en un subconjunto del conjunto factible: el conjunto de portafolios eficientes. Éste se caracteriza porque considera únicamente aquellos portafolios que generan un máximo nivel de rendimiento para un determinado nivel de riesgo; o bien, un mínimo riesgo para un rendimiento dado. Por tanto, si el objetivo del inversionista es maximizar la utilidad esperada, deberá considerar exclusivamente aquellos portafolios que son eficientes (Serrano, 1993: 24).

En este trabajo el diseño de portafolios eficientes se basa, primero, en la inclusión de un gran número de activos, y después, en que la correlación de los rendimientos entre los activos sea negativa. Esto, por un lado, permitirá diversificar el riesgo entre los diferentes activos y, por otro, se espera que el riesgo global del portafolio se reduzca en forma significativa. En este estudio, la elaboración de una cartera óptima de cultivos se basa fundamentalmente en estos dos principios.

\section{Resultados}

La información de los rendimientos de los diferentes cultivos considerados por grupo se extrajo de la base de datos del Sistema de Información Agropecuaria de Consulta (Siacon) de la Secretaría de Agricultura, Ganadería, Desarrollo Rural, Pesca y Alimentación (Sagarpa). En tanto que el programa computacional empleado para la estimación de la varianza de los rendimientos de cada grupo fue el Statistical Analysis System (SAS) en su versión $8^{\mathrm{a}}$ y Excel.

Los cultivos considerados, por grupo, en la estimación del riesgo y rendimiento se presentan en el cuadro 1.

Martínez y Díaz (2005: 75) mostraron que estos grupos, tomados en forma individual como portafolios de inversión, tanto frutales como hortalizas tienen rendimientos mayores respecto del grupo de cereales; sin embargo, también se encontró que este último grupo tenía un menor nivel de riesgo respecto de los frutales y hortalizas. Por lo que, si se observa la variable de rendimiento, resulta más atractivo invertir en frutales y hortalizas; aunque si se incorpora la variable de riesgo al análisis, esta decisión podría tomar otro sentido. 


\section{Cuadro 1 \\ Grupos de cultivos considerados ${ }^{6}$}

\begin{tabular}{|c|c|c|}
\hline Cereales & Frutales & Hortalizas \\
\hline Arroz (Oryza sativa) & Aguacate (Persea americana) & Cebolla (Allium sepa) \\
\hline Avena (Avena sativa) & Limón (Citrus uarantifolia) & Chile verde (Capsicum sp.) \\
\hline Cebada (Ordeum vulgare) & Mango (Manguifera indica) & Melón (Cucumis melo) \\
\hline Frijol (Phaseolus vulgare) & Manzana (Malus sp.) & $\begin{array}{l}\text { Papa (Solanum } \\
\text { tuberosum) }\end{array}$ \\
\hline Garbanzo (Cicer arietum) & Naranja (Citrus sinensis) & $\begin{array}{l}\text { Pepino (Ecballium } \\
\text { elaterium) }\end{array}$ \\
\hline Maíz (Zea mays) & Papaya (Carica papaya) & Sandía (Citrullus vulgaris) \\
\hline Sorgo (Sorghum vulgare) & Piña (Ananas comosus) & $\begin{array}{l}\text { Tomate rojo (Lico } \\
\text { persicum esculentum) }\end{array}$ \\
\hline Trigo (Triticum vulgare) & Plátano (Musa paradisiaca) & $\begin{array}{l}\text { Tomate verde (Physalis } \\
\text { exocarpa) }\end{array}$ \\
\hline
\end{tabular}

Cuando se seleccionaron los cultivos para construir una cartera óptima de inversión, se evaluaron diferentes opciones. Primero se consideraron los cultivos por grupos, lo que originó tres portafolios de inversión: cereales, frutales y hortalizas (cuadro 1). Posteriormente, de la totalidad de cultivos incluidos en los tres grupos, y de acuerdo con los principios de diversificación y correlación negativa de los rendimientos entre cultivos, se elaboraron tres portafolios alternativos (cuadro 2).

\section{Cuadro 2}

\section{Carteras de inversión de cultivos agrícolas para México}

\begin{tabular}{lll}
\hline Cartera 1 & Cartera 2 & Cartera 3 \\
\hline Sorgo & Sorgo & Avena \\
Aguacate & Trigo & Frijol \\
Mango & Aguacate & Sorgo \\
Manzana & Mango & Trigo \\
Naranja & Manzana & Aguacate \\
Piña & Naranja & Mango \\
Papa & Piña & Manzana \\
Sandía & Chile verde & Naranja \\
& Papa & Piña \\
& Sandía & Cebolla \\
& & Chile verde \\
& & Melón \\
& & Papa \\
& & Sandía \\
\hline
\end{tabular}

${ }^{6}$ Esta muestra de ocho artículos es representativa en el sentido de que los productos considerados constituyen más de $90 \%$ de la producción en el caso de los cereales y $80 \%$ en el caso de los frutales y hortalizas en el país, en relación con la producción total de su grupo (Sagarpa, 2005). 
En el portafolio 1 se integran los cultivos que presentaron mayor frecuencia de covarianza negativa de sus rendimientos respecto del total de cultivos. La cartera 2 sólo difiere por dos cultivos respecto de la cartera 1 , dichos cultivos también mostraron una alta frecuencia de covarianza negativa en sus rendimientos con el resto de los cultivos; sin embargo, dicha frecuencia fue ligeramente menor a la observada en los ocho cultivos de la cartera 1. Finalmente, la cartera 3 considera cuatro cultivos adicionales a los que presenta la cartera 2, esto, por un lado, atendiendo el principio de diversificación, y por otro, el de mayor frecuencia de covarianza negativa de los rendimientos entre cultivos. En el cuadro 3 se muestra la frecuencia de covarianza negativa de los rendimientos de cada cultivo respecto del total de éstos.

\section{Cuadro 3}

Frecuencia de covarianza negativa de los rendimientos de un cultivo específico respecto del total de cultivos

\begin{tabular}{lclclc}
\hline Cultivo & $\begin{array}{c}\text { Frecuenciade } \\
\text { covarianza } \\
\text { negativa }\end{array}$ & Cultivo & $\begin{array}{c}\text { Frecuenciade } \\
\text { covarianza } \\
\text { negativa }\end{array}$ & Cultivo & $\begin{array}{c}\text { Frecuenciade } \\
\text { covarianza } \\
\text { negativa }\end{array}$ \\
\hline Arroz & 4 & Aguacate & 14 & Cebolla & 6 \\
Avena & 6 & Limón & 6 & Chile verde & 7 \\
Cebada & 5 & Mango & 20 & Melón & 6 \\
Frijol & 6 & Manzana & 15 & Papa & 9 \\
Garbanzo & 4 & Naranja & 19 & Pepino & 4 \\
Maíz & 5 & Papaya & 5 & Sandía & 9 \\
Sorgo & 20 & Piña & 17 & Tomate rojo & 5 \\
Trigo & 7 & Plátano & 4 & Tomate verde & 4 \\
\hline
\end{tabular}

En este cuadro se muestra que el cultivo de sorgo tiene una fuerte asociación negativa de sus rendimientos respecto de 20 cultivos de los 24 considerados; en tanto que, para la naranja se observaron 19 resultados en este sentido y para la sandía 9. Aquí también se resume la frecuencia de covarianza negativa para el resto de los cultivos estudiados.

Una vez conformadas las carteras de inversión, se procedió a calcular el rendimiento y riesgo de cada una de ellas, a partir de las ecuaciones (3) y (8), respectivamente. Sin embargo, para no basar la elección de la cartera óptima únicamente en un solo resultado de riesgo y rendimiento, se realizaron 100 réplicas de estas variables para cada portafolio, considerando 100 combinaciones de ponderación (o asignaciones para $\mathrm{a}_{1}+\mathrm{a}_{2}+\ldots+\mathrm{a}_{\mathrm{N}}=1$ ). Debido a que no se trata de un estudio de simulación propiamente, 100 ré- 
plicas son suficientes para tener una buena aproximación a los valores medios tanto del riesgo como del rendimiento. ${ }^{7}$

Los resultados obtenidos, respecto del riesgo, para las carteras de cereales, frutales y hortalizas fueron de $0.1666,1.3460 \mathrm{y}$ 3.2395 , respectivamente, y en relación con los rendimientos, ${ }^{8}$ se observó 2.4118, 18.3035 y 17.0528, respectivamente. Esto es congruente con lo expuesto por Martínez y Díaz (2005: 75) en el sentido de que los cereales son el grupo con menos riesgo pero también con mucho menor nivel de rendimientos.

Por otra parte, los resultados del riesgo para las carteras 1, 2 y 3 son $1.3946,1.1175$ y 1.1280 , respectivamente; en tanto que los rendimientos fueron $14.5147,13.9387$ y 11.3915 , respectivamente. Por lo que es claro que la opción que representa la cartera 3 no es recomendable en el sentido de que muestra, por un lado, un mayor riesgo y un menor rendimiento respecto de la cartera 2 . Ahora bien, aunque la cartera 1 presenta mayor rendimiento que la 2, esta última implica un nivel de riesgo menor que la cartera 1 . De esta manera, puesto que elegir la cartera 1 en lugar de la 2 permitiría aumentar sólo marginalmente el rendimiento (de 13.9387 a 14.5147 ), $4.13 \%$, por otra parte, se incrementaría en forma sustancial el riesgo (de 1.1175 a 1.3946), $24.78 \%$. Por lo que, bajo la consideración de aversión al riesgo, la cartera de inversión óptima sería la 2.

Ahora, la cartera 2 también es mejor opción respecto de las de cereales, frutales y hortalizas. En cuanto a los cereales, es cierto que su nivel de riesgo es muy bajo pero su rendimiento también lo es. En relación con las hortalizas, no obstante que su rendimiento medio es mayor que el de la cartera 2 (17.0528 contra 13.9387), 22.34\%, el riesgo asociado a éstas es casi tres veces mayor al de la cartera 2 (3.2395 contra 1.1175). La cartera de frutales presentó un rendimiento medio de 18.3035, el cual es mayor en $31.31 \%$ al de la cartera 2 (de 13.9387); en tanto que el riesgo en frutales fue de 1.3460 , sólo 20.45 mayor al de la cartera 2 (de 1.1175). Esto último sugiere que la cartera de frutales es tan recomendable como la 2; sin embargo, como ésta no se construyó a partir de los principios de diversificación y la considera-

\footnotetext{
${ }^{7}$ Las 100 réplicas del riesgo y rendimiento involucran en cada caso portafolios de alto, mediano y bajo riesgo. La determinación de si un portafolio es de alto o bajo riesgo se define a partir de la asignación de altas ponderaciones a los activos con elevado rendimiento, en el primer caso, así como ponderaciones mínimas a estos mismos activos en el segundo.

${ }^{8}$ Las cifras de los rendimientos mostradas a partir de este párrafo, corresponden al rendimiento medio en toneladas de producto por hectárea (ton/ha).
} 
ción de activos con mayor frecuencia de covarianza negativa de sus rendimientos, esto hace que la cartera 2 sea mejor opción que la de frutales. Lo anterior se puede interpretar de la siguiente manera: elegir una cartera conformada únicamente por frutales en un país como México, no sólo sería inviable atendiendo la diversidad de condiciones agroecológicas de las diferentes regiones que lo conforman, también sería imposible tratar de reorientar la gran diversidad de cultivos agrícolas generados a únicamente los de frutales.

\section{Conclusiones}

A partir de los resultados de riesgo y rendimiento obtenidos para las carteras de inversión elaboradas en esta investigación, se observó que entre más elementos correlacionados en forma negativa en sus rendimientos integre un portafolio, tenderá a presentar menor nivel de riesgo para determinados grados de rendimiento.

De manera que es posible argumentar que la hipótesis establecida por Harry Markowitz -en el sentido de que aquellos portafolios que atienden los principios de diversificación e inclusión preferencial de los cultivos que se correlacionan en forma negativa en sus rendimientos, presentarán riesgos más bajos frente a niveles de rendimiento dados-, se mantiene en este trabajo.

De acuerdo con los resultados obtenidos en este estudio, es evidente que, para el país en su conjunto, mantener los niveles de producción de los diversos cultivos generados hasta ahora resulta poco eficiente en el sentido de que, por un lado, no se maximiza el nivel de rendimiento de los cultivos, y por el otro, tampoco se minimiza el grado de riesgo. De esta manera, la búsqueda de mayor eficiencia del sector agrícola involucra un replanteamiento del esquema de producción actual para tratar de transitar gradualmente a otro donde se incentive la producción de aquellos cultivos que permitan generar mayores rendimientos con poco riesgo, como los que se consideran en la cartera óptima de cultivos determinada en este estudio (cartera 2, cuadro 2).

No obstante que en este trabajo se obtuvieron conclusiones para México en su conjunto, debemos tomar en cuenta que las condiciones geográficas, climáticas, económicas y sociales -traducidas en escasez de recursos de los productores, poco conocimiento técnico, limitaciones de mercado que restringen las opciones de uso productivo de la tierra, así como insuficiencia de programas de fomento agrícola para la reconversión productiva (Rivera, 2004: 61), entre otras- son muy diversas. La propuesta 
de un posible replanteamiento gradual del esquema de producción agrícola actual, considera en buena medida la continuidad de la producción de una gran diversidad de cultivos, en virtud de que el portafolio óptimo incluye una amplia variedad de los mismos. Dicha propuesta es acorde con lo descrito por Quintana (2003: 8) en el sentido de que en el periodo 1991-2001 se observó un fuerte cambio en la geografía agrícola mexicana, pues mientras se reduce el área dedicada a granos básicos, hay un notable crecimiento en frutales y hortalizas; la superficie hortícola aumentó en 6\% y la frutícola en 21\%. Así, las adecuaciones que sugerimos tendrían que analizarse a partir de las condiciones de cada región para determinar en cuáles es viable realizar las conversiones convenientes de cultivos.

\section{Bibliografía}

González-Estrada, Adrián (2002), Dinámica de los cultivos básicos en la liberalización comercial de México: un modelo dinámico multisectorial de equilibrio general, México, Programa de Economía, INIFAP.

Markowitz, Harry (1952), "Portafolio Selection”, Journal of Finance, VII (1), pp. 77-91.

Markowitz, Harry (1991), Portfolio Selection, Malden Massachusetts, Blackwell-Publishers.

Martínez-Damián, Miguel A. (1999), "Evaluación del riesgo y evaluación de probabilidades: un análisis comparativo", Agrociencia, 33(1), Colegio de Postgraduados, México, pp.119-122.

Martínez-Damián, Miguel A. y Miguel Angel Díaz-Carreño (2005), “Comparación de la varianza estimada de los índices de precios al consumo de cereales, frutales y hortalizas en México, 1980-2000”, Análisis Económico, xx (45), Universidad Autónoma Metropolitana-Azcapotzalco, México, pp.75-88.

Mood, Alexander M., Franklin A. Graybill y Duane C. Boes (1974), Introduction to the Theory of Statistics, , New York, McGraw Hill. 
Quintana S., Víctor M. (2003), "El círculo vicioso del Tratado de Libre Comercio de América del Norte: la amarga experiencia mexicana en el agro a partir del TLCAN", Deslinde, 33, Centro de Estudios del Trabajo (Cedetrabajo), Bogotá, Colombia, pp.26-38.

Rivera-Herrerón, G. (2004), "La agricultura del Estado de México: una visión de largo plazo”, en P. Mejía Reyes (coord.), La economía del Estado de México, hacia una agenda de investigación, México, El Colegio Mexiquense, pp. 31-72.

Ross, Sheldon M. (2002), Probabilidad y estadística para ingenieros, México, McGraw Hill.

Sachs, Jeffrey D. y Felipe Larraín D. (1994), Macroeconomía en la economía global, México, Prentice Hall Hispanoamericana.

Sagarpa (Secretaría de Agricultura, Ganadería, Desarrollo Rural, Pesca y Alimentación) (2005), Centro de Estadística Agropecuaria (CEA). Sistema de Información Agropecuaria de Consulta (Siacon), versión 1.1, México, Sagarpa.

Sánchez, Carlos (2001), Valor en riesgo y otras aproximaciones, México, SEI Investments de México.

SAS Institute (1999), Statistical Analysis System, versión 8a , programa computacional estadístico.

Serrano-Espinosa, Julio (1993), Introducción a las carteras de inversión, México, Instituto Tecnológico Autónomo de México-IMA-SSP.

Siller-Cepeda, Jorge H. (2004), Situación actual de la industria hortofrutícola en México, México, Centro de Investigación en Alimentación y Desarrollo, A. c., Unidad Culiacán.

Suárez, Blanca (1995), "La apertura comercial y los cultivos de exportación: las frutas y hortalizas”, Análisis Económico, XII(27), Universidad Autónoma Metropolitana-Azcapotzalco, México, pp. 55-73. 
Tobin, James (1958). "Liquidity preference as behavior towards risk", Review of Economic Studies, February (67), Cowles Fundation paper 118. pp. 65-86.

Miguel Ángel Díaz Carreño. Es doctor en ciencias con especialidad en economía por el Colegio de Postgraduados. Es profesorinvestigador en la Facultad de Economía de la Universidad Autónoma del Estado de México. Su línea de investigación se centra en economía de los sectores productivos. Sus publicaciones más recientes son: "Pruebas no paramétricas para procesos Poisson no homogéneos", Agrociencia, 37(1) pp. 21-31 (2003); "Comparación de la varianza estimada de los índices de precios al consumo de cereales, frutas y hortalizas en México, 1980-2002”, Análisis Económico, $\mathrm{xx}(45)$, pp. 75 -88 (2005); "Estudio comparativo del nivel de riesgo entre los cultivos de granos, frutales y hortalizas, 1980-2003”, Ciencia Ergo Sum, 13(2), Universidad Autónoma del Estado de México, pp. 143-148 (2006).

Rafael Juárez Toledo. Es maestro en economía por la Facultad de Economía de la Universidad Autónoma del Estado de México. Actualmente es coordinador de Investigación y Estudios Avanzados de dicha facultad. Su línea de investigación se centra en economía de los sectores productivos.

María del Carmen Gómez Chagoya. Maestra en economía por la Facultad de Economía de la Universidad Autónoma del Estado de México. Actualmente se desempeña como profesora-investigadora en la facultad mencionada. Su línea de investigación se centra en economía de los sectores productivos. 